\title{
Femtogram Detection of Explosive Nitroaromatics: Fluoranthene-Based Fluorescent Chemosensors
}

\author{
N. Venkatramaiah, Shiv Kumar, and Satish Patil*[a]
}

\begin{abstract}
Herein we report a novel fluoranthene-based fluorescent fluorophore 7,10-bis(4-bromophenyl)-8,9-bis[4-(hexyloxy)phenyl]fluoranthene $\quad\left(\mathbf{S}_{\mathbf{3}}\right)$ and its remarkable properties in applications of explosive detection. The sensitivity towards the detection of nitroaromatics (NACs) was evaluated through fluorescence quenching in solution, vapor, and contact mode approaches. The contact mode approach using thin-layer silica chromatographic plates exhibited a femtogram
\end{abstract}

$\left(1.15 \mathrm{fg} \mathrm{cm}^{-2}\right)$ detection limit for trinitrotoluene (TNT) and picric acid (PA), whereas the solution-phase quenching showed PA detection at the 2-20 ppb level. Fluorescence lifetime measurements revealed that the quenching is static in nature and the quenching process is fully reversible.

Keywords: explosives $\cdot$ femtochemistry $\cdot$ fluorescence $\cdot$ nitroaromatics • sensors
Binding energies between model binding sites of the $\mathbf{S}_{\mathbf{3}}$ and analyte compounds reveal that analyte molecules enter into the cavity created by substituted phenyl rings of fluoranthene and are stabilized by strong intermolecular interactions with alkyl chains. It is anticipated that the sensor $\mathbf{S}_{\mathbf{3}}$ could be a promising material for the construction of portable optical devices for the detection of onsite explosive nitroaromatics.

\section{Introduction}

Conjugated polycyclic aromatic hydrocarbons (PAHs) like fluoranthene and fluorene have received considerable attention for their potential applications in optoelectronics and fluorescent chemosensors. ${ }^{[1-3]}$ The high thermal stability and anomalous fluorescence characters of fluoranthene serve as a basic skeleton for the preparation of a blue-light emitter for organic light-emitting diodes (OLEDs). ${ }^{[4,5]}$ Fluorescent conjugated polymers have been extensively used for the detection of highly explosive nitroaromatic compounds (NACs) for national security, environmental protection, remediation of munitions sites, and forensic investigations. ${ }^{[6-9]}$ A variety of conjugated polymers such as $\operatorname{poly}(p$-phenylene vinylenes), polysilanes, polycarbazoles, and polymeric porphyrins have been used as sensing materials for the highly sensitive and selective detection towards 2,4,6-trinitrotoluene (TNT) and picric acid (PA) in solution and vapor phases. ${ }^{[10-14]}$ Various analytical methods such as fluorescence and gas chromatography coupled with mass spectrometry, surface-enhanced Raman spectrometry, and electrochemical methods have been developed for the detection of NACs. ${ }^{[15-20]}$ In particular, the fluorescence-quenching technique has been widely employed for many polymers with

[a] Dr. N. Venkatramaiah, S. Kumar, Prof. S. Patil

Solid State and Structural Chemistry Unit

Indian Institute of Science, Bangalore 560012 (India)

Tel: (+91) 80-22932651

Fax: (+91)80-23601310

E-mail: satish@sscu.iisc.ernet.in

Supporting information for this article is available on the WWW under http://dx.doi.org/10.1002/chem.201201764. high sensitivity and selectivity. ${ }^{[21,22]}$ Efforts have been made to employ fluorescent inorganic nanoparticles like silica, $\mathrm{ZnS}$, and CdSe quantum dots in the detection of explosive compounds through luminescence quenching. ${ }^{[21,23]}$ Swager et al. have developed amplifying fluorescent pentiptycenederived poly ( $p$-phenylene vinylene) (PPV) and poly(phenylene ethynylene) (PPE)-based polymer films for the detection of TNT and 2,4-dinitrotoluene (DNT) vapors at subpart-per-trillion (ppt) levels. ${ }^{[7,11]}$ Recently, Ajayaghosh et al. reported a subfemtogram level of TNT detection with perfluoroarene-based gelator coated on disposable paper strips. ${ }^{[24]}$ However, most of the research work to date has been directed towards the design of novel fluorescent conjugated polymers. The majority of these polymers suffer intrinsic interferences from other electron acceptors and require laborious synthetic work that is time consuming and inefficient, which leads to difficulties in upscaling, high throughput screening, and practical applications. Therefore the development of novel sensory materials with superior performance and detection methods for traces of nitroaromatics are at the heart of current interest. Small-moleculebased fluorescent chromophores with a rich $\pi$-electron-donating character have received greater attention than conjugated polymers on account of their better sensitivity. A series of small-molecule fluorophores such as pyrene, coumarins, purpurin, fluorescein, and some other indicator dyes were employed for the detection of NACs because of their long-lived excited-states. ${ }^{[24-27]}$ Recently, Zyryanov et al. have developed 1,4-diarylpentiptycene-based small molecules for the visual detection of DNT $(0.18 \mathrm{ppm})$ and TNT (7.7 ppb). ${ }^{[28]}$ Costa et al. have reported substituted $p$-phenylene ethynylene trimer (CALIX-PET and TBP-PET)-based 
materials for the detection of NACs. ${ }^{[29]}$ To emphasize the importance and exploration of fluoranthene as an excellent donor material for better sensitivity and high detection towards explosive nitroaromatics, ${ }^{[30]}$ herein we report a new $\pi$ conjugated fluoranthene derivative 7,10-bis(4-bromophenyl)-8,9-bis[4-(hexyloxy)phenyl]fluoranthene $\left(\mathbf{S}_{\mathbf{3}}\right)$ as a sensor material for the detection of explosive NACs. The presence of flexible long alkyl chains on fluoranthene forms strong intermolecular interactions with analytes, and sterically crowded phenyl rings on the backbone of $\mathbf{S}_{\mathbf{3}}$ hinder aggregation and excimer formation.

\section{Results and Discussion}

Compound $\mathbf{S}_{\mathbf{3}}$ was synthesized by a three-step process and is shown in Scheme 1. First, 1,2-bis[4-(hexyloxy)phenyl]ethyne was synthesized by a Sonogashira cross-coupling reaction. In

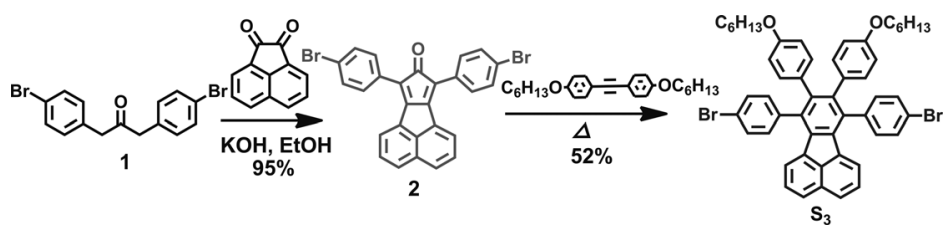

Scheme 1. Schematic representation of the synthesis of fluoroanthene derivative $\mathbf{S}_{3}$.

a second step, 7,9-bis(4-bromophenyl)-8H-cyclopenta[a]acenaphthylen-8-one (2) was synthesized by heating acenaphthenedione and 1,3-bis(4-bromophenyl)propan-2-one to reflux in ethanol for $15 \mathrm{~min}$, which afforded compound $\mathbf{2}$ in $95 \%$ yield. Finally, a Diels-Alder reaction was carried out between 1,2-bis[4-(hexyloxy)phenyl]ethyne and 7,9-bis(4-bromophenyl)-8H-cyclopenta $[a]$ acenaphthylen-8-one in $1,2,4$-trichlorobenzene at $220^{\circ} \mathrm{C}$ in a sealed glass pressure tube to afford compound $\mathbf{S}_{\mathbf{3}}$ in $52 \%$ yield.

$\mathrm{X}$-ray-quality single crystals were obtained by a slow solvent evaporation method in a dichloromethane/ethanol mixture at ambient temperature. The single-crystal X-ray structure of $\mathbf{S}_{\mathbf{3}}$ (Figure 1a) revealed a monoclinic space group, $P 21 / c$, with $a=12.976, b=22.970, c=14.783 \AA ; \alpha=90, \beta=$
105.20, $\gamma=90^{\circ}$; and $Z=4$. The pertinent crystallographic data of $\mathbf{S}_{\mathbf{3}}$ is provided in the Supporting Information (Table S1). In the solid state, the two phenyl rings at C7 and C8 are twisted by torsional angles of 61.16 and $60.25^{\circ}$, and the other two phenyl rings at $\mathrm{C} 15$ and $\mathrm{C} 22$ are twisted by 6.34 and $11.4 \AA$, respectively. This twisting of the phenyl rings leads to a very weak $\pi-\pi$ interaction of $6.527 \AA$, and the central fluoranthene core has an almost planar structure. The supramolecular arrangement of $\mathbf{S}_{\mathbf{3}}$ along the $a b$ plane (Figure 1b) shows the formation of Z-shaped structural arrangements in which one of the fluoranthene units enters into the cavity of two alkyl chains of the other fluoranthene unit and is stabilized by strong $\mathrm{C}-\mathrm{H} \cdots \pi$ hydrogen-bonding interactions. We observed the presence of two nonequivalent $\mathrm{C}-\mathrm{H} \cdots \pi$ interactions at the $\mathrm{C} 47$ and $\mathrm{C} 52$ atoms by 2.372 and $2.781 \AA$. The formation of different types of packing arrangements in the solid state provoked our interest in their detection of NACs.

Figure 2 shows the absorption and emission spectra in solution and thin film on a quartz plate. The absorption spectra of $\mathbf{S}_{\mathbf{3}}$ in chloroform exhibit two major bands with absorption maxima at 303 and $374 \mathrm{~nm}$, which arise from the $\pi \rightarrow \pi^{*}$ transitions. The emission spectra show strong blue fluorescence at $475 \mathrm{~nm}$ with a Stokes shift of $101 \mathrm{~nm}$. The absorption and emission spectra of $\mathbf{S}_{\mathbf{3}}$ in thin film are quite similar to that of the solution spectra. However, we observed the split in the emission pattern for thin film at 458 and $486 \mathrm{~nm}$, which indicates the strong perturbation in the vibronic

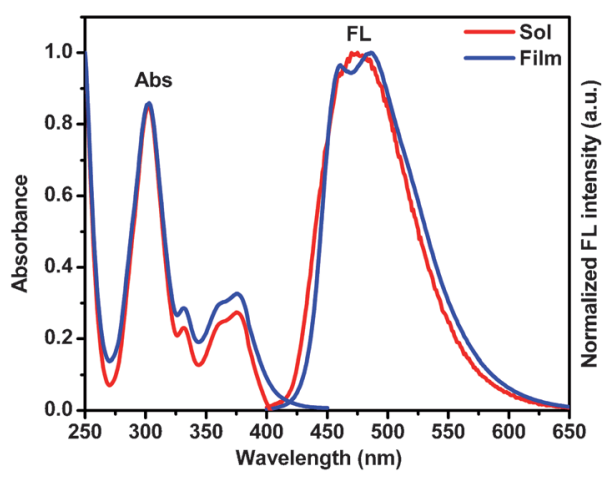

Figure 2. The optical absorption and fluorescence spectra of $\mathbf{S}_{\mathbf{3}}$ in chloroform and in thin film.
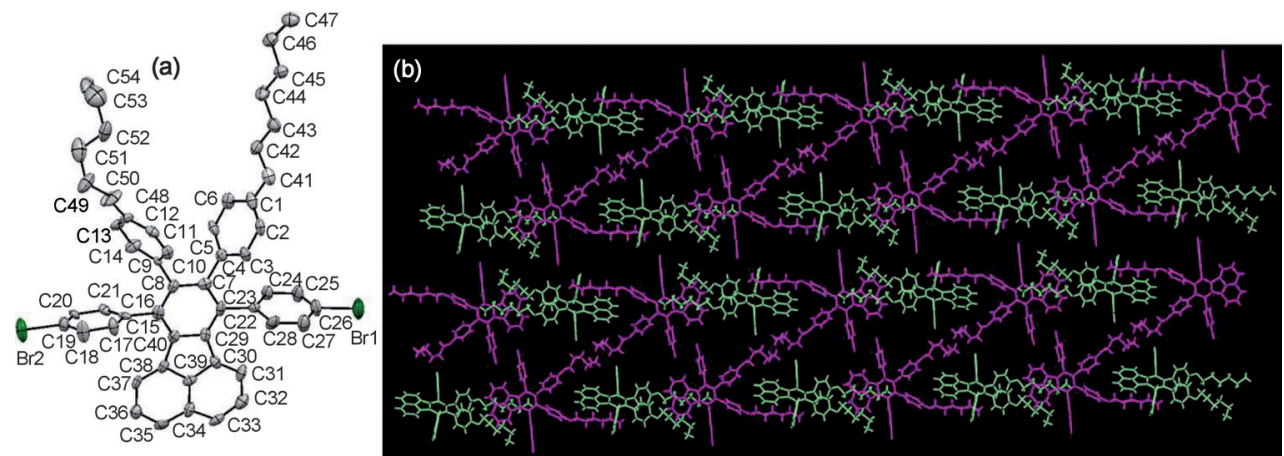

Figure 1. a) Single-crystal X-ray structure of $\mathbf{S}_{\mathbf{3}}$. The hydrogen atoms are omitted for clarity. b) The supramolecular arrangement of $\mathbf{S}_{\mathbf{3}}$ along the $a b$ plane. 
states due to the heavy-atom effect in the excited state. The optical bandgaps were calculated to be 3.084 and $3.017 \mathrm{eV}$ in solution and thin film, respectively. Based on the spectral pattern of the absorption and fluorescence, we can infer that $\mathbf{S}_{\mathbf{3}}$ exhibits weak $\pi-\pi$ stacking interactions, and that the arrangement of the molecules in the thin film is quite similar to the solid-state packing. The fluorescence lifetime of $\mathbf{S}_{\mathbf{3}}$ in chloroform was found to be approximately $11.37 \mathrm{~ns}$ by the time-correlated single-photon counting (TCSPC) method. The quantum yield $(\Phi)$ of $\mathbf{S}_{\mathbf{3}}$ is $25 \%$ in chloroform and $44 \%$ in hexane.

To explore the potential applications of $\mathbf{S}_{\mathbf{3}}$ as a sensory material for the detection of NACs, such as PA, TNT, DNT, 2,4-dinitrophenol (DNP), and common interfering agents like 1,4-dichlorobenzene (DCB), benzoquinone (BQ), and other analytes, fluorescence titration experiments were carried out in ethanol. Figure $3 \mathrm{a}$ shows the fluorescence quenching behavior of $\mathbf{S}_{\mathbf{3}}$ upon gradual addition of a $0.1 \mathrm{~mm}$ solution of PA. The fluorescence intensity was found to decrease significantly as a function of concentration, and $80 \%$ quenching was observed after the addition of picric acid $(10 \mu \mathrm{L}, 0.1 \mathrm{~mm})$. Further, fluorescence quenching was analyzed by the Stern-Volmer (SV) equation $I_{0} / I=1+K_{\mathrm{sv}}[\mathrm{PA}]$, in which $I_{0}$ and $I$ are the fluorescence intensities of $\mathbf{S}_{\mathbf{3}}$ in the presence and absence of PA, respectively, and $K_{\mathrm{sv}}$ is the Stern-Volmer constant.

On the basis of quenching, a linear SV plot was obtained with a rate constant of $1.24 \times 10^{5} \mathrm{M}^{-1}$, which was found to be much higher than those of other fluorescent chemosensors toward PA. ${ }^{[36-38]}$ On the other hand, we have not observed any significant changes in the fluorescence quenching behavior for other NACs at this concentration level. However, fluorescence was efficiently quenched when $\mathbf{S}_{\mathbf{3}}$ was treated with gradual addition of NACs $(10 \mu \mathrm{L})$ at a concentration of $5 \times 10^{-3} \mathrm{M}$ (Figure S12 in the Supporting Information). On the basis of the SV plots (Figure $3 \mathrm{~b}$ ), $K_{\mathrm{sv}}$ was found to be $1828 \mathrm{M}^{-1}$ for TNT, $1772 \mathrm{M}^{-1}$ for DNT, and $178 \mathrm{M}^{-1}$ for BQ. This suggests that sensor $\mathbf{S}_{\mathbf{3}}$ is highly sensitive towards the detection of PA in ethanol. The appearance of a linear SV plot for all analytes is due to the formation of static or dynamic quenching processes. However, static quenching involves the formation of a ground-state nonfluorescent charge-transfer (CT) complex, whereas dynamic quenching involves the excited-state electron transfer from fluorophore to oxidative quencher. To understand this phenomenon, fluorescence lifetime studies were carried out at different concentrations of PA. The fluorescence lifetime of $\mathbf{S}_{\mathbf{3}}$ was found to be invariant with concentration of PA (Figure 3c), which suggests that quenching follows static phenomena through ground-state charge-transfer complex formation. This was further confirmed by ${ }^{1} \mathrm{H}$ NMR spectroscopy and electronic absorption titration of $\mathbf{S}_{\mathbf{3}}$ with different concentrations of picric acid (Figure S13 in the Supporting Information). Upon addition of PA to $\mathbf{S}_{\mathbf{3}}$, the ${ }^{1} \mathrm{H}$ NMR spectra shows a gradual upfield shift in the doublet proton at $\delta=7.75$ to $7.72 \mathrm{ppm}$ for a 1:1 molar ratio and reaches equilibrium. This indicates that the gradual upfield shift is attributed to a
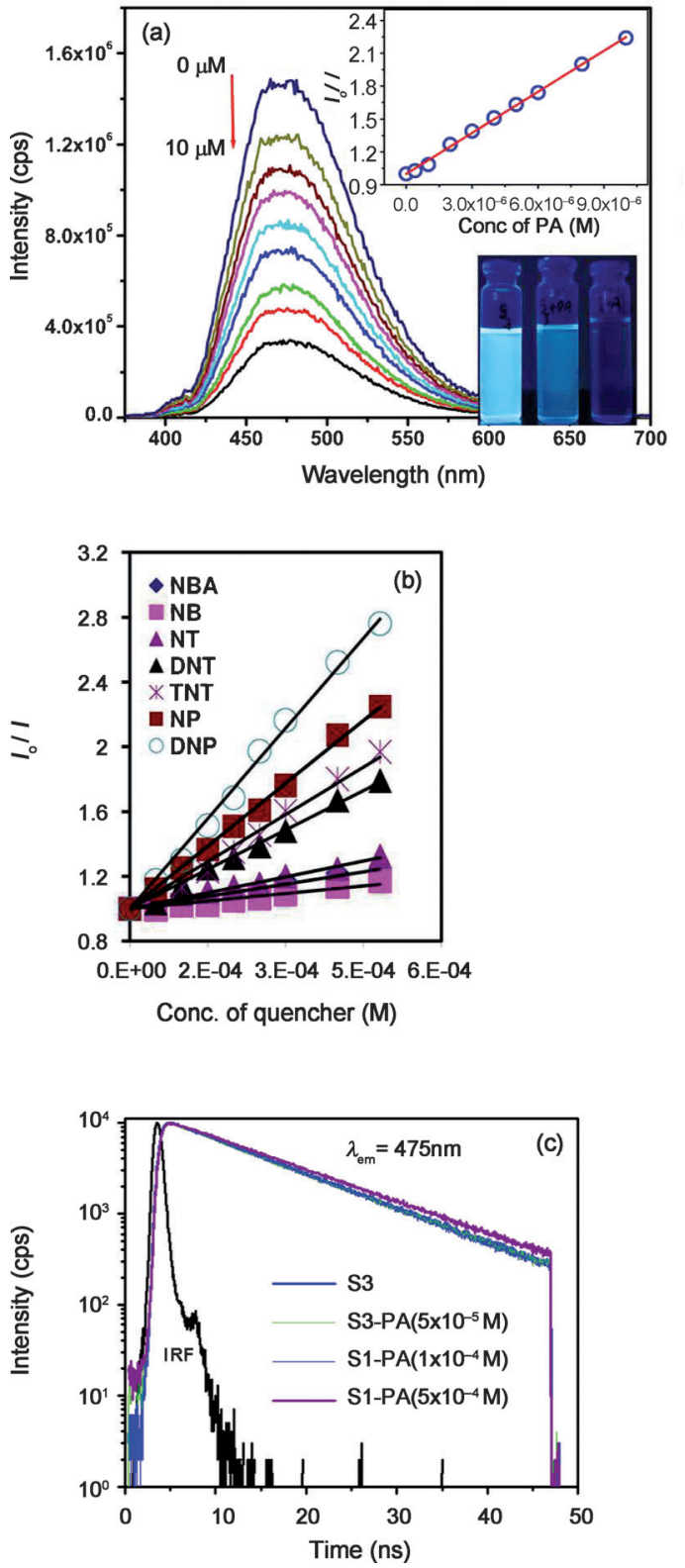

Figure 3. a) Fluorescence quenching of $\mathbf{S}_{\mathbf{3}}\left(1 \times 10^{-6} \mathrm{M}\right)$ upon addition of different concentrations of PA $\left(1 \times 10^{-4} \mathrm{M}\right)$ in ethanol $\left(\lambda_{\mathrm{ex}}=370 \mathrm{~nm}\right)$. The inset shows the Stern-Volmer plot for different concentrations of PA. The inset also shows changes in the fluorescence intensity after addition of $10 \mu \mathrm{M}$ of PA to $\mathbf{S}_{3}$. b) The Stern-Volmer plot for different concentrations of various nitroaromatics with $\mathbf{S}_{3}$. c) Fluorescence lifetime measurements $\left(\lambda_{\mathrm{em}}=470 \mathrm{~nm}\right)$ for different concentrations of PA with $\mathbf{S}_{\mathbf{3}}$ in ethanol.

bound charge-transfer complex. The formation of a chargetransfer complex was further confirmed by electronic absorption spectra. The spectrophotometric titration of $\mathbf{S}_{\mathbf{3}}(5 \times$ $10^{-6} \mathrm{M}$ ) with different concentrations of picric acid (PA) in ethanol is shown in Figure S14 in the Supporting Information. Upon gradual addition of PA to $\mathbf{S}_{\mathbf{3}}$, there was a slight increase in the absorption maxima at $303 \mathrm{~nm}$ along with the formation of a new broad absorption peak at $424 \mathrm{~nm}$. The intensity of the new absorption peak increased gradually as 
the concentration of PA increased. This indicated the formation of a donor-acceptor complex between the $\pi$-electronrich $\mathbf{S}_{\mathbf{3}}$ fluorophore and picric acid. Similar results were observed upon treatment of $\mathbf{S}_{\mathbf{3}}$ with other electron-deficient nitroaromatics.

The molecular interaction with other electron-deficient compounds such as 2,3-dichloro-5,6-dicyano-1,4-benzoquinone (DDQ), 1,2-acenaphthylenedione (ANQ), 1-chloroanthraquinone (1-ClAQ), and 9,10-phenanthrenequinone (PAQ) was also investigated. We observed the quenching of fluorescence intensity at $475 \mathrm{~nm}$ with the appearance of a new emission peak at $390 \mathrm{~nm}$. The intensity of the new emission peak increased and showed nonlinear behavior in the SV plot, which suggests that $\mathbf{S}_{\mathbf{3}}$ follows both static as well as dynamic quenching processes (Figure S15 in the Supporting Information). Figure 4 shows the histogram of quenching ef-

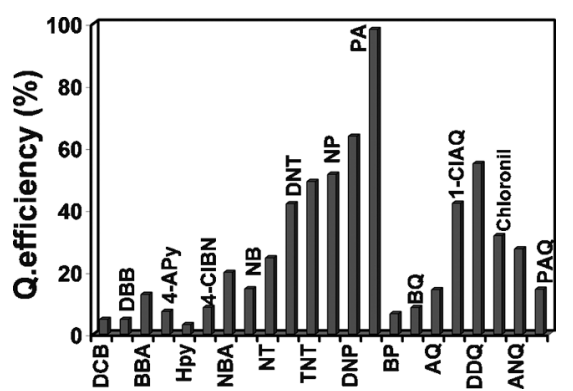

Figure 4. The fluorescence-quenching efficiency of $\mathbf{S}_{\mathbf{3}}$ upon addition of different analyte solutions at $25^{\circ} \mathrm{C}$.

ficiency for different nitroaromatics and other electron-deficient interferants. Common analytes such as dichlorobenzene (DCB), pyridine (Py), and 4-bromobenzoic acid (BBA) have little effect on the fluorescence intensity. On the other hand, electron-deficient nitroaromatics brought about the greatest quenching depending upon the number of nitro substituents present on the toluene and phenol moiety. PA exhibits the highest quenching response at parts-per-billion levels (2-20 ppb), whereas DNT and TNT show a level of detection of approximately $1.1 \mathrm{ppm}$ in solution.

To further understand the mode of interaction and unusual sensitivity of PA with $\mathbf{S}_{\mathbf{3}}$, density functional calculations (DFT) were carried out at the B3LYP/6-31g* level using the Gaussian 03 package. ${ }^{[35]}$ From the optimized geometry, it was observed that the HOMO orbital has large overlap of electron density localized on the phenyl rings of the alkyl chains and the fluoranthene backbone, whereas the electron density in the LUMO is largely localized on the fluoranthene unit (Figure 5). The LUMO energy of $\mathbf{S}_{\mathbf{3}}(-1.76 \mathrm{eV})$ lies higher in energy than PA $(-3.89 \mathrm{eV})$. With a view to obtaining strong evidence for the fluorescence-quenching response and the type of interaction, we optimized the geometries of the fluorophore-analyte complexes. On the basis of the single-crystal analysis, the analytes form two types of fluorophore-analyte complexes in which analyte molecules enter the cavity created by substituted phenyl rings of fluo-

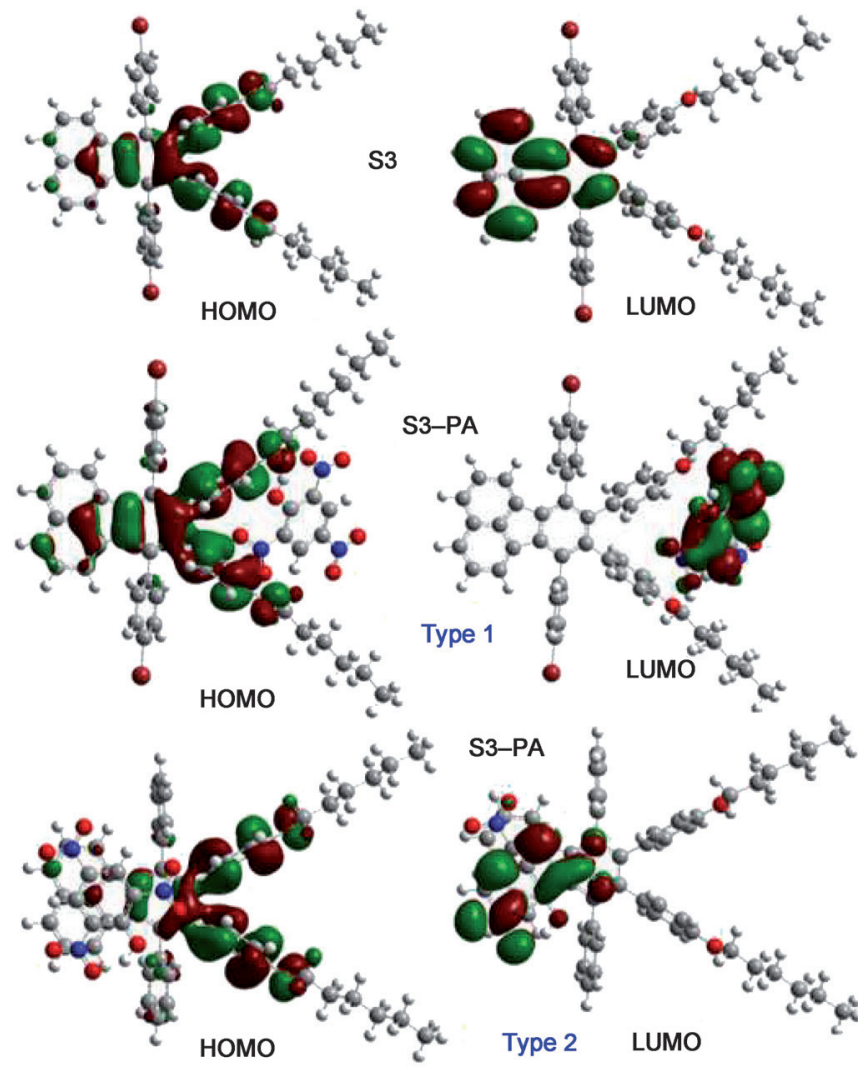

Figure 5. Electron-density profiles of $\mathbf{S}_{\mathbf{3}}$ and $\mathbf{S}_{\mathbf{3}}-\mathrm{PA}$ complexes with the B3LYP/6-31g* basis set.

ranthene and are stabilized by strong intermolecular interactions (type 1). The other possibility is to form a cofacial stacked geometry and strong intermolecular $\pi-\pi$ interactions (type 2). A representative example of $\mathbf{S}_{\mathbf{3}}-\mathrm{PA}$ complexes is shown Figure 5.

The total energy of type $1 \mathbf{S}_{\mathbf{3}}$-PA complex is found to be less than type 2 by $14.82 \mathrm{kcal} \mathrm{mol}^{-1}$. This indicates that the alkyl chains of $\mathbf{S}_{\mathbf{3}}$ have a strong energetic affinity toward picric acid due to the proximity of the electron density and two of the nitro groups of picric acid oriented towards the alkyl chains by 2.57 and $2.55 \AA$, whereas the other nitro and alcohol groups are oriented out of the cavity. The calculated binding energy for the type $1 \mathbf{S}_{3}-\mathrm{PA}$ complex is $32123.29 \mathrm{kcalmol}^{-1}$, which is energetically higher than type $2\left(31228.79 \mathrm{kcal} \mathrm{mol}^{-1}\right)$. Figure 5 shows the frontier molecular orbital pictures of $\mathbf{S}_{\mathbf{3}}$ and $\mathbf{S}_{\mathbf{3}}-\mathrm{PA}$ complexes. For type 1, the HOMO orbitals are localized on phenyl and fluoranthene units similar to $\mathbf{S}_{\mathbf{3}}$ and the LUMO orbitals are localized on picric acid. This facilitates a strong electron transfer from the excited state of $\mathbf{S}_{\mathbf{3}}$ to PA in the fluorescencequenching process in type 1 rather than in type 2 . Similar results were observed for $\mathbf{S}_{\mathbf{3}}$-TNT complexes and are shown in the Supporting Information (Figure S19).

To demonstrate the potential utility of the material towards the detection of nitroaromatics in the vapor phase, a thin film of $\mathbf{S}_{\mathbf{3}}$ was fabricated by spin-coating techniques on 
quartz substrate at a rate of $2000 \mathrm{rpm}$ and a concentration of $1 \mathrm{mg}$ per $0.2 \mathrm{~mL}$ in chloroform. The film was annealed at $50{ }^{\circ} \mathrm{C}$ for $2 \mathrm{~h}$. The fluorescence response of the films to the vapors of NACs was ascertained by inserting the film into sealed vials that contained solid explosives at room temperature. Figure 6 shows the time-dependent fluorescence

a)

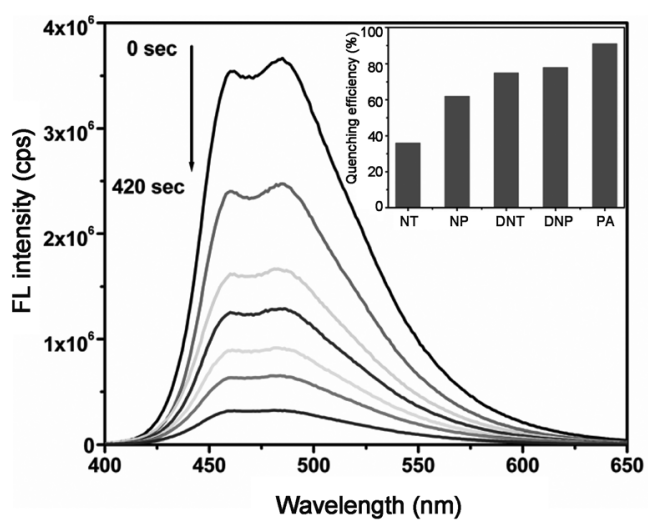

b)

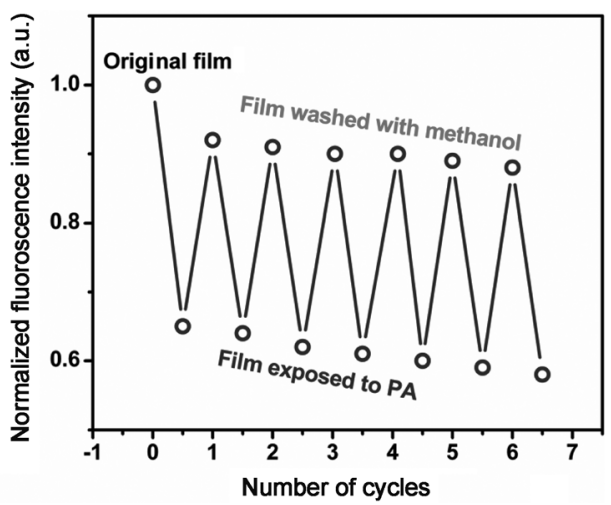

Figure 6. a) Time-dependent emission spectra of $\mathbf{S}_{\mathbf{3}}$ thin film upon exposure to the saturated vapors PA at $60 \mathrm{~s}$ time intervals. The inset shows the fluorescence-quenching efficiency for different saturated vapors of nitroaromatics. b) The reversibility of $\mathbf{S}_{\mathbf{3}}$ thin film to the saturated vapors of PA after $120 \mathrm{~s}$ of exposure time.

quenching of $\mathbf{S}_{\mathbf{3}}$ film upon exposure to saturated vapors of PA. As expected, the fluorescence intensity was found to decrease as the time of exposure elapsed. The initial emission intensity was significantly reduced by $33 \%$ after $60 \mathrm{~s}$ of exposure and then by $66 \%$ after $180 \mathrm{~s}$. Upon continuous exposure, $92 \%$ quenching was observed after $420 \mathrm{~s}$ of exposure time and ultimately reached its equilibrium. This indicates that $\mathbf{S}_{\mathbf{3}}$ film exhibits a fast response for the detection of PA at ppb levels. It is interesting to note that the film of $\mathbf{S}_{\mathbf{3}}$ also shows response to the vapors of DNT, DNP, NP, and NT (Figure S17 in the Supporting Information). However, the extent of quenching efficiency was found to be smaller than the saturated vapors of PA and is shown in the inset of Figure $6 \mathrm{a}$. The variations in the quenching behavior might be due to differences in partial pressure, redox potential of the analytes, electron-accepting capability of $\mathbf{S}_{\mathbf{3}}$, and adsorptive affinity of film toward the NACs. The trapping of PA vapors into the cavities created by alkoxy chains of phenyl rings and the formation of strong intermolecular interactions with alkyl chains of $\mathbf{S}_{\mathbf{3}}$ results in high quenching performance. Figure $6 \mathrm{~b}$ shows the reversibility of the $\mathbf{S}_{\mathbf{3}}$ film towards the detection of PA. The film was exposed to saturated vapors of PA at room temperature for $120 \mathrm{~s}$ and the emission spectrum was recorded. The film was washed with methanol and dried at $45^{\circ} \mathrm{C}$ under vacuum, the emission spectrum was recorded, and the whole process was repeated. The results show that the initial fluorescence intensity was significantly retained after several washings, thereby indicating a high photostability of the film. A quenching efficiency of $88 \%$ was observed even after 6 cycles of exposure to PA vapors.

It is well known that the vapor pressures of nitroaromatics such as PA and TNT are extremely high when sensing the proximity of explosives. Therefore, we carried out a surface sensing approach to test the viability by coating $\mathbf{S}_{\mathbf{3}}$ on thinlayer silica chromatographic plates. The visual change in the fluorescence intensity makes it applicable to onsite instant detection of explosives.

To test this possibility, we prepared different concentrations of various NACs. Each solution $(5 \mu \mathrm{L})$ was spotted onto the $\mathbf{S}_{\mathbf{3}}$-coated thin-layer silica plate to give a spot area of approximately $0.2 \mathrm{~cm}^{2}$. The visual fluorescence response to different analytes at different concentration levels is shown in Figure 7a. The fluorescence intensity was found to decrease as a function of concentration, and the minimum amount of TNT and PA detectable by the naked eye was as low as $5 \mu \mathrm{L}$ of $1 \times 10^{-13} \mathrm{M}$ solution with a detection limit of approximately $1.15 \mathrm{fg} \mathrm{cm}^{-2}$. Figure $7 \mathrm{~b}$ shows the fluorescence-quenching pattern of $\mathbf{S}_{3}$-coated TLC plates for different concentrations of TNT solution. We observed 12 and $17 \%$ quenching for $1 \times 10^{-13} \mathrm{M}$ solutions of TNT and PA, respectively. A comparison of the fluorescence-quenching efficiency for different nitroaromatic analytes is shown in Figure 7c. The quenching effect was found to be higher for TNT and PA than the other nitroaromatics.

\section{Conclusion}

In conclusion, we have developed a novel fluoranthenebased small-molecule fluorescent chemosensor for femtogram detection of TNT and picric acid. The unique supramolecular arrangement of the fluoranthene molecules demonstrates that nitroaromatics enter into the cavity created by the substituted phenyl rings and are stabilized by strong intermolecular interaction with the alkyl chains, thereby resulting in efficient fluorescence quenching. The sensor $\mathbf{S}_{\mathbf{3}}$ is highly sensitive and is able to detect picric acid at the ppb level, whereas it shows ppm-level sensitivity toward TNT in ethanol. The vapor-phase fluorescence-quenching study revealed that the fluorescence of $\mathbf{S}_{\mathbf{3}}$ film is efficiently quenched by DNT and PA vapors. The quenching process is 
creasing the electron-donating strength for better sensitivity are ongoing in our laboratory.

\section{Experimental Section}

All the nitroaromatics such as PA, TNT, DNP, DNT, NP, NT, and NB were obtained from Sigma Aldrich and were used without further purification.

Caution: PA, TNT, and other NACs used in the present study are highly explosive and should be handled only in small quantities. ${ }^{1} \mathrm{H}$ and ${ }^{13} \mathrm{C}$ NMR spectra were recorded using Bruker 400 and $100 \mathrm{MHz}$ NMR spectrometers and were calibrated by using TMS as an internal reference. Chemical shifts are reported in parts per million (ppm). The UV/ Vis spectra were recorded using a Perkin-Elmer (Lambda 35) UV/Vis spectrometer. All the absorption spectra in solution were recorded in $\mathrm{CHCl}_{3}$ (concentration of $1 \times 10^{-5} \mathrm{~mol} \mathrm{~L}^{-1}$ ) and the spectra in the solid state were recorded from films of compounds spin-coated on quartz substrate. Steady-state fluorescence emission studies were carried out using a Spex FluoroLog-3 spectrofluorometer (Jobin-Yvon Inc.). The fluorescence-quenching experiments were carried out in a quartz cell in ethanol medium. The solid-state fluorescence-quenching performance in the vapor phase was tested under saturated vapors of NACs and data was collected from the front face of the film. Time-resolved fluorescence measurements were carried out with time-correlated single-photon counting (TCSPC) with nanosecond LED $(295 \mathrm{~nm})$ at a repetition rate of $1 \mathrm{MHz}$ used to excite the samples. Single-crystal X-ray diffraction datasets were collected using an Oxford Xcalibur (Mova) diffractometer equipped with a EOS CCD detector that used $\mathrm{Mo}_{\mathrm{K} a}$ radiation $(\lambda=$ $0.71073 \AA) \cdot{ }^{[31]}$ The crystal was maintained at the desired temperature during data collection using the Oxford Instruments Cryojet-HT controller. ${ }^{[32]}$ All structures were solved by direct methods using SHELXS-97 and refined against $F^{2}$ using SHELXL-97. Hydrogen atoms were fixed geometrically and refined isotropically. The WinGX package was used for refinement and production of data tables and ORTEP-3 for structure visualization and making molecular representations that showed the ellipsoids at $30 \%$ probability level. ${ }^{[33]}$ Analysis of the hydrogen-bonding and $\pi-\pi$ interactions was carried out using PLATON, and packing diagrams were generated by using MERCURY. ${ }^{[34]}$ All quantum chemical calculations were carried out in Gaussian 03 by using the B3LYP hybrid function with $6-31 \mathrm{~g} *$ basis set. ${ }^{[35]}$

Synthesis procedure: Compound 1,2-bis[4-(hexyloxy)phenyl]ethyne was synthesized by a Sonogashira cross-coupling reaction and the experimental details are provided in the Supporting Information (Scheme S1).

Synthesis of 1,3-bis(4-bromophenyl)propan-2-one (1): A solution of 4bromophenylacetic acid $(5.0 \mathrm{~g}, 23 \mathrm{mmol})$ in dichloromethane was added dropwise to a mechanically stirred solution of dicyclohexylcarbodiimde (DCC; $2.4 \mathrm{~g}, 12 \mathrm{mmol}$ ) and $N, N$-dimethylaminopyridine (DMAP; $0.71 \mathrm{~g}$, $0.58 \mathrm{mmol})$ in dichloromethane $(40 \mathrm{~mL})$. The reaction was allowed to proceed overnight under an argon atmosphere at room temperature. The reaction mixture was filtered to remove dicyclohexylurea, and the filtrate was subjected to rotary evaporation. The product was purified by column chromatography with $10 \%$ ethyl acetate/hexane as eluent (53\% yield). ${ }^{1} \mathrm{H}$ NMR $\left(400 \mathrm{MHz}, \mathrm{CDCl}_{3}, 25^{\circ} \mathrm{C}, \mathrm{TMS}\right): \delta=7.43\left(\mathrm{~d},{ }^{3} J(\mathrm{H}, \mathrm{H})=6.8 \mathrm{~Hz}\right.$, $4 \mathrm{H}), \quad 7.00\left(\mathrm{~d},{ }^{3} J(\mathrm{H}, \mathrm{H})=6.8 \mathrm{~Hz}, 4 \mathrm{H}\right), \quad 3.67 \mathrm{ppm}(\mathrm{s}, 4 \mathrm{H}) ;{ }^{13} \mathrm{C} \mathrm{NMR}$ $\left(100 \mathrm{MHz}, \mathrm{CDCl}_{3}, 25^{\circ} \mathrm{C}, \mathrm{TMS}\right): \delta=204.20,132.58,131.83,131.21,121.24$, $48.43 \mathrm{ppm}$.

Synthesis of 7,9-bis(4-bromophenyl)-8H-cyclopenta $[a]$ acenaphthylen-8one (2): Compound 2 was synthesized by double Knoevenagel condensation reaction between 1,3-bis(4-bromophenyl)propan-2-one $(\mathbf{1} ; 1.87 \mathrm{~g}$, $5.08 \mathrm{mmol})$ and acenaphthylene-1,2-dione $(0.92 \mathrm{~g}, 5.08 \mathrm{mmol})$ in ethanol $(50 \mathrm{~mL})$. A solution of $\mathrm{KOH}(0.095 \mathrm{~g})$ in ethanol $(2 \mathrm{~mL})$ was added dropwise, and the reaction mixture was heated to reflux for $15 \mathrm{~min}$ under vigorous stirring. The reaction mixture was cooled to $0^{\circ} \mathrm{C}$, and the dark green solid was filtered, washed with ethanol, and dried (95\% yield). The obtained compound was insoluble in common solvents. highly reversible and static in nature. Femtogram detection levels for PA and TNT were achieved by the contact-mode approach on thin-layer silica chromatographic plates. Efforts towards the structural modification of fluoranthene by in- 
Synthesis of 7,10-bis(4-bromophenyl)-8,9-bis[4-(hexyloxy)phenyl]fluoranthene $\left(\mathbf{S}_{3}\right)$ : A $15 \mathrm{~mL}$ pressure glass tube was charged with 7,10-bis(4-bromophenyl)- $8 H$-cyclopenta[ $a]$ acenaphthylen- 8 -one $(0.102 \mathrm{~g}, 0.2 \mathrm{mmol})$ and 1,2-bis[4-(hexyloxy)phenyl]ethyne $(0.075 \mathrm{~g}, 0.28 \mathrm{mmol})$, to which a minimal amount (approximately $1.5 \mathrm{~mL}$ ) of 1,2,4-trichlorobenzene was added. It was degassed by bubbling argon and sealed. The heterogeneous purple-black solution was heated to $220^{\circ} \mathrm{C}$ with vigorous stirring until it became homogeneous and took on a transparent greenish-yellow color after $24 \mathrm{~h}$. The reaction mixture was cooled in an ice bath and diluted with hexane to precipitate the crude product, which was collected by filtration and washed with copious amounts of ethanol. The resulting pale yellow solid was then purified by passing it through a silica gel column with $5 \%$ ethyl acetate/hexane mixture as eluent to yield pure $\mathbf{S}_{\mathbf{3}}(92 \mathrm{mg}$, $52 \%$ yield). ${ }^{1} \mathrm{H}$ NMR (400 MHz, $\mathrm{CDCl}_{3}, 25^{\circ} \mathrm{C}$, TMS): $\delta=7.73$ (d, ${ }^{3} J$ $(\mathrm{H}, \mathrm{H})=8.1 \mathrm{~Hz}, 2 \mathrm{H}), 7.46\left(\mathrm{~d},{ }^{3} J(\mathrm{H}, \mathrm{H})=8.2 \mathrm{~Hz}, 4 \mathrm{H}\right), 7.34\left(\mathrm{t},{ }^{3} J(\mathrm{H}, \mathrm{H})=\right.$ $7.6 \mathrm{~Hz}, 2 \mathrm{H}), 7.17\left(\mathrm{~d},{ }^{3} J(\mathrm{H}, \mathrm{H})=8.2 \mathrm{~Hz}, 4 \mathrm{H}\right), 6.73\left(\mathrm{~d},{ }^{3} J(\mathrm{H}, \mathrm{H})=8.5 \mathrm{~Hz}\right.$, $4 \mathrm{H}), 6.65\left(\mathrm{~d},{ }^{3} J(\mathrm{H}, \mathrm{H})=7.1 \mathrm{~Hz}, 2 \mathrm{H}\right), 6.46\left(\mathrm{~d},{ }^{3} J(\mathrm{H}, \mathrm{H})=8.5 \mathrm{~Hz}, 4 \mathrm{H}\right), 3.78$ $\left(\mathrm{t},{ }^{3} J(\mathrm{H}, \mathrm{H})=6.7 \mathrm{~Hz}, 4 \mathrm{H}\right), 1.67\left(\mathrm{dd}, J_{1}(\mathrm{H}, \mathrm{H})=14.4, J_{2}(\mathrm{H}, \mathrm{H})=6.9 \mathrm{~Hz}, 4 \mathrm{H}\right)$, $1.44-1.36(\mathrm{~m}, 4 \mathrm{H}), 1.31(\mathrm{~s}, 8 \mathrm{H}), 0.89 \mathrm{ppm}\left(\mathrm{t},{ }^{3} J(\mathrm{H}, \mathrm{H})=6.7 \mathrm{~Hz}, 6 \mathrm{H}\right)$; ${ }^{13} \mathrm{C} \mathrm{NMR}\left(100 \mathrm{MHz}, \mathrm{CDCl}_{3}, 25^{\circ} \mathrm{C}, \mathrm{TMS}\right): \delta=156.76,140.69,138.98$, $136.38,136.24,136.12,133.12,132.06,131.81,131.66,131.41,129.66$, 127.66, 126.69, 123.03, 120.96, 113.04, 67.70, 31.59, 29.18, 25.64, 22.53, $13.98 \mathrm{ppm}$; elemental analysis calcd $(\%)$ for $\mathrm{C}_{52} \mathrm{H}_{48} \mathrm{Br}_{2} \mathrm{O}_{2}: \mathrm{C} 72.22, \mathrm{H}$ 5.59; found: C 72.14, H 5.74

\section{Acknowledgements}

The authors would like to acknowledge the NMR Research Centre, IISc for NMR spectra. We would also like to thank Mr. K. Durga Prasad and Prof. T. N. Guru Row for single-crystal XRD analysis. A financial grant from DST and DIT is gratefully acknowledged.

[1] R. C. Chiechi, R. J. Tseng, F. Marchioni, Y. Yang, F. Wudl, Adv. Mater. 2006, 18, 325.

[2] S. J. Toal, W. C. Trogler, J. Mater. Chem. 2006, 16, 2871.

[3] H. F. Leng, W. H. Wu, J. React. Funct. Polym. 2012, 72, 206.

[4] A. Goel, V. Kumar, S. Chaurasia, M. Rawat, R. Prasad, R. S. Anand, J. Org. Chem. 2010, 75, 3656.

[5] Q. Yan, Y. Zhou, B.-B. Ni, Y. Ma, J. Wang, J. Pei, Y. Cao, J. Org. Chem. 2008, 73, 5328.

[6] Y. Salinas, R. M. Máñez, M. D. Marcos, F. Sancenón, A. M. Costero, M. Parra, S. Gil, Chem. Soc. Rev. 2012, 41, 1261.

[7] J. S. Yang, T. M. Swager, J. Am. Chem. Soc. 1998, 120, 11864.

[8] M. E. Germain, M. J. Knapp, Chem. Soc. Rev. 2009, 38, 2543.

[9] Q. L. Fang, J. L. Geng, B. H. Liu, D. M. Gao, F. Li, Z. Y. Wang, G. J. Guan, Z. P. Zhang, Chem. Eur. J. 2009, 15, 11507.

[10] G. M. Murry, B. M. Arnold, D. S. Lawrence, US Patent 2001077664, 2001

[11] J. S. Yang, T. M. Swager, J. Am. Chem. Soc. 1998, 120, 5321.

[12] Y. Dong, J. W. Y. Lam, A. Qin, Z. Li, J. Liu, J. Sun, Y. Dong, B. Z. Tang, Chem. Phys. Lett. 2007, 446, 124.

[13] H. Nie, Y. Zhao, M. Zhang, Y. Ma, M. Baumgarten, K. Müllen, Chem. Commun. 2011, 47, 1234.

[14] H. Nie, G. Sun, M. Zhang, M. Baumgarten, K. Müllen, J. Mater. Chem. 2012, 22, 2129.

[15] R. Hodyss, J. L. Beauchamp, Anal. Chem. 2005, 77, 3607.
[16] T. H. Kim, B. Y. Lee, J. Jaworski, K. Yokoyama, W. J. Chung, E. Wang, S. Hong, A. Majumdar, S. W. Lee, ACS Nano 2011, 5, 2824

[17] E. Schramm, J. Hölzer, M. Pütz, R. Schulte-Ladbeck, R. Schultze M. Sklorz, A. Ulrich, J. Wieser, R. Zimmermann, Anal. Bioanal. Chem. 2009, 395, 1795.

[18] M. Stępień, B. Donnio, J. L. Sessler, Angew. Chem. 2007, 119, 1453; Angew. Chem. Int. Ed. 2007, 46, 1431.

[19] Y. B. Zhang, Y. W. Tan, H. L. Stormer, P. Kim, Nature 2005, 438, 201

[20] J. M. Sylvia, J. A. Janni, J. D. Klein, K. M. Spencer, Anal. Chem. 2000, 72,5834

[21] A. Rose, Z. Zhu, C. F. Madigan, T. M. Swager, V. Bulovic, Nature 2005, 434, 876

[22] Y. Long, H. Chen, Y. Yang, H. Wang, Y. Yang, N. Li, K. Li, J. Pei, F. Liu, Macromolecules 2009, 42, 6501.

[23] L. H. Jin, S. M. Li, Y. H. Cho, Biosens. Bioelectron. 2012, 33, 284.

[24] K. K. Kartha, S. S. Babu, S. Srinivasan, A. Ajayaghosh, J. Am. Chem. Soc. 2012, 134, 4834.

[25] G. He, N. Yan, J. Yang, H. Wang, L. Ding, S. Yin, Y. Fang, Macromolecules 2011, 44, 4759.

[26] M. S. Meaney, V. McGuffin, Anal. Bioanal. Chem. 2008, 391, 2557.

[27] A. Lapat, L. Székelyhidi, I. Hornyák, Biomed. Chromatogr. 1997, $11,102$.

[28] G. Zyryanov, M. A. Palacios, P. Anzenbacher Jr, Org. Lett. 2008, 10, 3681.

[29] A. I. Costa, J. V. Prata, Sensor. Actuat. B: Chem. 2012, 161, 702.

[30] N. Venkatramaiah, S. Kumar, S. Patil, Chem. Commun. 2012, 48, 5007.

[31] Oxford Diffraction (2009). CrysAlis CCD and CrysAlisPro RED, Version 1.171.33.34d, Oxford Diffraction Ltd., Abingdon, Oxfordshire, U.K..

[32] G. Sheldrick, Acta Crystallogr. Sect. A 2008, 64, 112.

[33] L. Farrugia, J. Appl. Crystallogr. 1997, 30, 565.

[34] L. Farrugia, J. Appl. Crystallogr. 1999, 32, 837.

[35] Gaussian 03, Revision C.02, M. J. Frisch, G. W. Trucks, H. B. Schlegel, G. E. Scuseria, M. A. Robb, J. R. Cheeseman, J. A. Montgomer y, Jr., T. Vreven, K. N. Kudin, J. C. Burant, J. M. Millam, S. S. Iyengar, J. Tomasi, V. Barone, B. Mennucci, M. Cossi, G. Scalmani, N Rega, G. A. Petersson, H. Nakatsuji, M. Hada, M. Ehara, K Toyota, R. Fukuda, J. Hasegawa, M. Ishida, T. Nakajima, Y. Honda O. Kitao, H. Nakai, M. Klene, X. Li, J. E. Knox, H. P. Hratchian, J. B. Cross, V. Bakken, C. Adamo, J. Jaramillo, R. Gomperts, R. E. Stratmann, O. Yazyev, A. J. Austin, R. Cammi, C. Pomelli, J. W. Ochterski, P. Y. Ayala, K. Morokuma, G. A. Voth, P. Salvador, J. J. Dannenberg, V. G. Zakrzewski, S. Dapprich, A. D. Daniels, M. C Strain, O. Farkas, D. K. Malick, A. D. Rabuck, K. Raghavachari, J. B. Foresman, J. V. Ortiz, Q. Cui, A. G. Baboul, S. Clifford, J. Cioslowski, B. B. Stefanov, G. Liu, A. Liashenko, P. Piskorz, I. Komaromi, R. L. Martin, D. J. Fox, T. Keith, M. A. Al-Laham, C. Y. Peng, A. Nanayakkara, M. Challacombe, P. M. W. Gill, B. Johnson, W. Chen, M. W. Wong, C. Gonzalez, J. A. Pople, Gaussian, Inc., Wallingford CT, 2004.

[36] S. Shanmugaraju, D. Samanta, B. Gole, P. S. Mukherjee, Dalton Trans. 2011, 40, 12333 .

[37] H. Sohn, R. M. Calhoun, M. J. Sailor, W. C. Trogler, Angew. Chem. 2001, 113, 2162; Angew. Chem. Int. Ed. 2001, 40, 2104.

[38] G. He, H. Peng, T. Liu, M. Yang, Y. Zhang, Y. Fang, J. Mater. Chem. 2009, 19, 7347

Received: May 18, 2012 Published online: September 27, 2012 